\title{
Three-dimensional superresolution in metamaterial slab lenses: Experiment and theory
}

\author{
F. Mesa \\ Department de Física Aplicada I. Universidad de Sevilla, 41012-Sevilla, Spain \\ M. J. Freire, R. Marqués, and J. D. Baena \\ Department de Electrónica y Electromagnetismo, Universidad de Sevilla, 41012-Sevilla, Spain
}

(Received 29 September 2005; published 27 December 2005)

\begin{abstract}
This paper presents a theoretical and experimental study on the viability of obtaining two- and threedimensional superresolution (i.e., resolution overcoming the diffraction limit for all directions in space) by means of metamaterial slab lenses. Although the source field cannot be actually reproduced at the back side of the lens with superresolution in all space directions, the matching capabilities of metamaterial slabs does make possible the detection of images with three-dimensional superresolution. This imaging takes place because of the coupling between the evanescent space harmonic components of the field generated at both the source and the detector.
\end{abstract}

DOI: 10.1103/PhysRevB.72.235117

PACS number(s): 42.30.Wb, 41.20.Jb, 73.20.Mf, 78.20.Ci

\section{INTRODUCTION}

It is well known from the early works of Veselago $^{1}$ that a slab made of a left-handed medium will focus the electromagnetic energy coming from a point source to another point located at the opposite side of the slab. An experimental confirmation of this focusing of energy has been reported by Houck et al. ${ }^{2}$ Subsequent works ${ }^{3-5}$ have shown that, under some circumstances, metamaterial lenses made of lefthanded media can produce images at certain planes with a resolution beyond the classical diffraction limit, or "superresolution imaging" (SRI). This SRI has been attributed to an amplification, inside the lens, of the evanescent space Fourier harmonics (SFHs) coming from the source. ${ }^{3}$ It has been also discussed ${ }^{6}$ that this process gives rise to fields that decay exponentially from the lens toward the image, which causes superresolution to take place only in planes parallel to the slab interfaces. In the direction perpendicular to the lens, a strong decay of the field is observed, and thus, a threedimensional (3D) picture of the source cannot be directly obtained from the field distribution at the back side of the lens. In other words, superresolution in the transverse directions is obtained at the price of a drastic loss of resolution in the longitudinal direction. This fact has been experimentally corroborated by recently reported field measurements, ${ }^{4}$ where the field growth from the image plane to the "super lens" can be clearly appreciated. Other experimental results also lead to the same conclusion, showing that images "of finite depth"5 cannot be directly obtained from field measurements in SRI experiments.

Following the seminal paper of Pendry, ${ }^{3}$ other superresolution devices have been proposed making use of slabs of negative permittivity, ${ }^{7,8}$ ferrite slabs, ${ }^{9}$ coupled planar polariton-resonant metasurfaces, ${ }^{10}$ and a pair of coupled magnetoinductive surfaces. ${ }^{11}$ In spite of the different physical nature of their constitutive elements, all these structures present several common characteristics. In this way, the above well-defined family of imaging devices can be characterized by: (i) the presence of planar slabs, (ii) the distance between the source and the image planes is always $2 d$ (where $d$ is the slab width), and (iii) the imaging properties are based on the amplification of evanescent SFHs inside the slab. Consequently, the imaging properties of Pendry's left-handed slab lens are also expected to be shared by these devices. ${ }^{6}$ Nevertheless, some experimental results recently reported by some of the authors ${ }^{11}$ have suggested the possibility of obtaining superresolution in the longitudinal direction in one of the devices mentioned above. In these experiments, a 3D map of a pointlike source (i.e., a source of subwavelength size) was obtained at the image side of the lens. In other words, three-dimensional superresolution imaging (3D-SRI) was obtained. The aim of the present contribution is to provide the general theory underlying this 3D-SRI. It will be shown that 3D-SRI of pointlike sources is actually a general property of the aforementioned family of devices, provided that the appropriate detection procedure is followed.

\section{ANALYSIS}

The main difference between SRI and standard imaging processes is that, in the former, the information for the image formation is carried out by evanescent SFHs, ${ }^{3}$ which can support subwavelength information, unlike the propagative SFHs responsible of the conventional imaging, which can only transport overwavelength information. Since evanescent fields cannot carry power and any image measurement requires some power transmission, it is apparent that the detection procedure has to somehow "create" a traveling power flux. This combined effect should then perturb, substantially, the fields around the detector in a way similar to that found in the tunneling effect. As is well known, the tunneling of power is due to the excitation of a pair of evanescent electromagnetic waves, whose interference gives rise to a nonvanishing flux of power. "Perfect tunneling" of power in a waveguide filled by a metamaterial has been recently reported by some of the authors. ${ }^{12}$ In this work it is shown that maximum tunneling of power in a setup with identical input and output waveguides is achieved when the output is placed at a distance from the input equal to that from the source to 


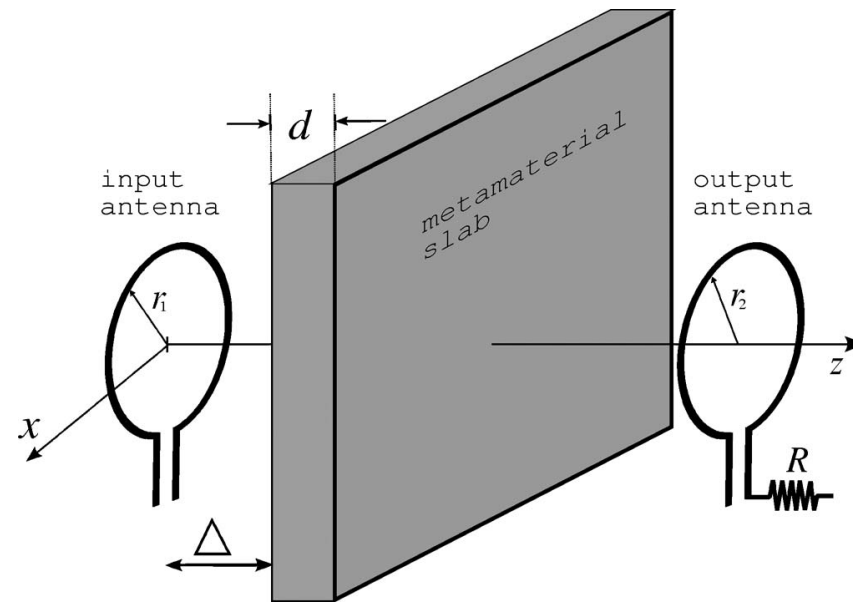

FIG. 1. Geometry of a metamaterial lens. The source is a loop antenna located at $z=0$. The detector is also an identical loop antenna. The lens is formed either by a left-handed metamaterial slab or by another planar device that produces SRI through amplification of evanescent harmonics. The output antenna can eventually be loaded with microwave resistors in order to minimize field perturbation.

the image in a metamaterial superlens. This suggests that a similar effect could take place in a metamaterial superlens, provided that a detector identical to the source is used for the measurements. In the following, how to take advantage of such an effect in order to obtain 3D-SRI with metamaterial slabs will be shown.

The present study starts with the canonical problem of the formation of images by a left-handed slab of thickness $d$ characterized by $\epsilon / \epsilon_{0}=\mu / \mu_{0}=-1+i \delta$, where $\delta \ll 1$ accounts for the necessary losses factor to avoid the divergence of field integrals. ${ }^{13-15}$ In our study, and following a usual procedure in microwave SRI experiments, the source will be an antenna (specifically, a loop antenna) whose plane is located parallel to the slab interfaces as shown in Fig. 1. The field beyond the lens will be scanned by an output antenna that plays the role of detector. The source employed here is equivalent to an homogeneous surface distribution of magnetic dipoles given by

$$
M_{s}= \begin{cases}I_{0} e^{-i \omega t}, & \text { inside the loop } \\ 0, & \text { outside },\end{cases}
$$

where $I$ denotes the amplitude of the imposed time-harmonic current in the loop. The computation of the longitudinal magnetic field $H_{z}$ at the image side of the slab $(z>\Delta+d)$ can be carried out by means of the following double-inverse Fourier transform:

$$
H_{z}(x, y, z)=\frac{1}{4 \pi^{2}} \iint \mathrm{d} k_{x} \mathrm{~d} k_{y} \widetilde{G}\left(k_{x}, k_{y} ; z\right) \tilde{M}_{s}\left(k_{x}, k_{y}\right) e^{i\left(k_{x} x+k_{y} y\right)},
$$

where $\widetilde{G}\left(k_{x}, k_{y} ; z\right)$ is the Fourier transform of the Green's function of the structure under study and $\tilde{M}_{s}\left(k_{x}, k_{y} ; z\right)$, the Fourier transform of the spatial surface distribution of mag-

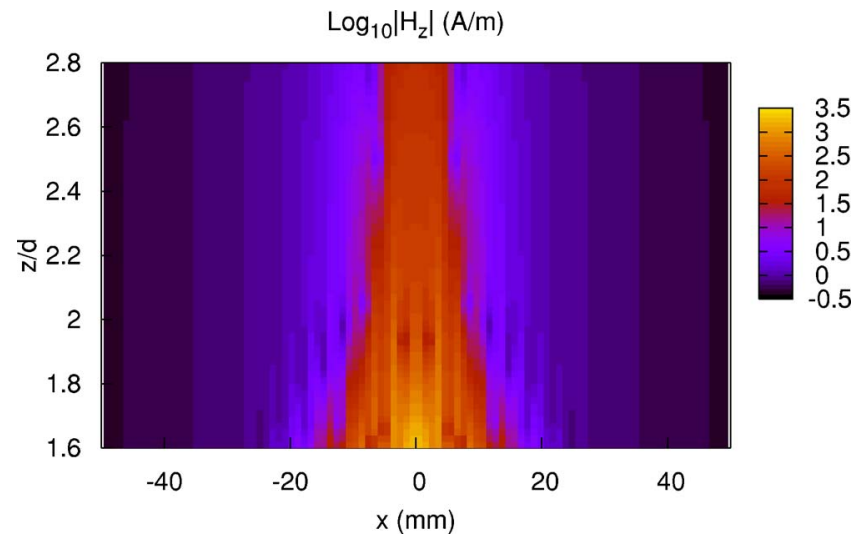

FIG. 2. (Color online) Map of $\log _{10}\left|H_{z}\right|$ corresponding to the field generated at the back side of the lens by a source loop antenna shown in Fig. 1. The slab is made of a left-handed medium of thickness $d=4 \mathrm{~mm}$ with $\epsilon / \epsilon_{0}=\mu / \mu_{0}=-1+i 0.001$, and is separated by a distance $\Delta=d / 2$ from the source. The source is considered, for simplicity, a circular loop antenna of radius $r_{1}=5 \mathrm{~mm}$. The wire radius is of $0.2 \mathrm{~mm}$. The operation frequency is $3 \mathrm{GHz}$.

netic dipoles. After applying the duality principle to expression (6) in Ref. 15 and taking $\Delta=d / 2$, the Fourier transform of the Green's function is found to be

$$
\widetilde{G}\left(k_{x}, k_{y} ; z\right)=\frac{-\left(k_{x}^{2}+k_{y}^{2}\right) 2 \beta e^{-i \beta_{0}(z+d)}}{\omega\left\{\left(\beta \mu_{0}-\beta_{0} \mu\right)^{2} e^{i \beta d}-\left(\beta \mu_{0}+\beta_{0} \mu\right)^{2} e^{-i \beta d}\right\}},
$$

where

$$
\begin{gathered}
\beta=\sqrt{\omega^{2} \varepsilon \mu-k_{x}^{2}-k_{y}^{2}} \\
\beta_{0}=\sqrt{\omega^{2} \varepsilon_{0} \mu_{0}-k_{x}^{2}-k_{y}^{2}} .
\end{gathered}
$$

Since $E_{z}=0$ in the present case, all the remaining field components can be deduced from $H_{z}{ }^{15}$

The numerical computation of (2) provides the map depicted in Fig. 2 for the magnitude of $H_{z}$ in the $(x-z)$ plane at the back side of the lens. It should be noted that, given that the operation frequency is $3 \mathrm{GHz}\left(\lambda_{0}=100 \mathrm{~mm}\right)$ and that $d$ and $\Delta$ are taken much less than the free-space wavelength, the ray model approximation cannot be employed here to obtain the fields in the considered region (in other words, we are dealing with near fields and therefore in a SRI situation). According to previous discussions, Fig. 2 shows that the field magnitude has a strong decay along the $z$ direction, so that no information about the location of the source in the longitudinal direction can be extracted from the field distribution. A more detailed picture of the field distribution at two planes $z=(2 \pm \delta) d$ is plotted in Fig. 3 (with $\delta$ set to 0.3 ) and compared to the field amplitude near the source at $z= \pm \delta d$ (the field distributions at $z=\delta d$ and $z=-\delta d$ are identical). As is expected from the properties of the metamaterial slab, the field distribution at the $z=(2+\delta) d$ plane is almost identical to that at the $z=\delta d$ plane, thus confirming that the $z=\delta d$ and the $z=(2+\delta) d$ planes are, in fact, conjugate planes. On the contrary, the field distribution at the $z=(2-\delta) d$ plane substan- 


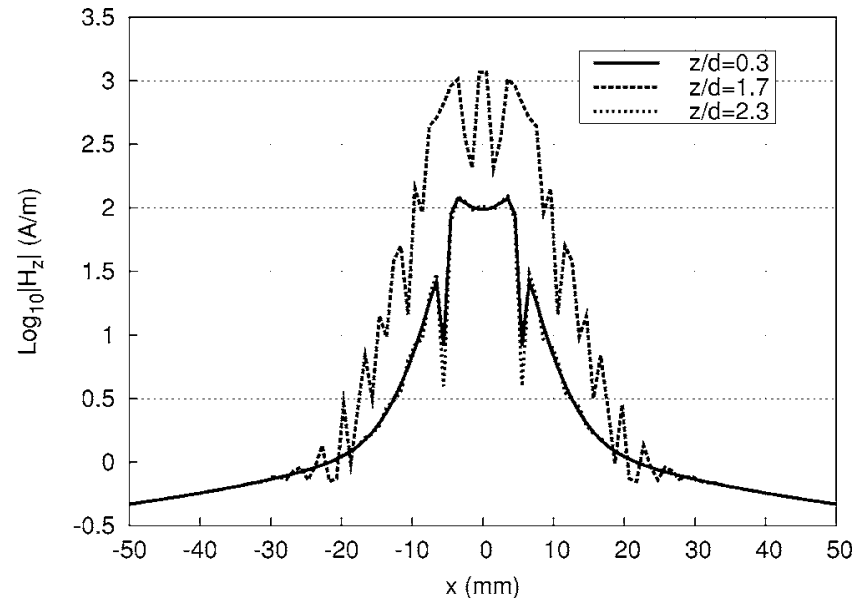

FIG. 3. Plot of the $\log _{10}\left|H_{z}\right|$ at different $z$-planes of Fig. 1. The slab and the source loop antenna are as in Fig. 2.

tially differs in magnitude from the field distribution at the $z=-\delta d$ plane, near the source. These facts corroborate the aforementioned discussions: the superresolution in the transverse directions (the lateral dimensions of the source and the image are approximately one-tenth of the wavelength) is compensated by an almost complete loss of resolution in the longitudinal direction.

The problem of the measurement of the image in the SRI setup under study is next considered. In the microwave range, the image detection is performed by measuring the transmission coefficient between a source antenna and a receiving antenna, which is scanned in the image side of the lens. ${ }^{2,4,5}$ For simplicity, the receiving (or output) antenna is assumed to be identical to the input antenna employed as source. The transmission coefficient is measured by connecting the input antenna to a wave generator via a waveguide, and the output one to a detector through another identical waveguide (more details of this measurement setup are reported in Ref. 11). In this approach, the metamaterial slab should be viewed as a matching device, ${ }^{16}$ whose transmission coefficient $t$ (or $S_{21}$ in the usual microwave terminology) is given by ${ }^{17}$

$$
t=\frac{2 Z_{12} Z_{0}}{\left(Z_{11}+Z_{0}\right)\left(Z_{22}+Z_{0}\right)-Z_{12}^{2}},
$$

where $Z_{i j}$ are the elements of the impedance matrix for the system formed by the two antennas and the left-handed slab, and $Z_{0}$ is the characteristic impedance of the input and output waveguide. In order to measure a superresolution image, the size of the loop antennas has to be smaller than the freespace wavelength, which additionally would make the real part of $Z_{i j}$ (the radiation resistance ${ }^{17}$ ) be negligible with respect to its imaginary part; namely, $Z_{i j} \simeq-i \omega L_{i j}$, where $L_{i j}$ is the inductance matrix of the system. The diagonal terms of the inductance matrix correspond to the inductances of a single-loop antenna faced to the left-handed slab. However, in the "perfect lens" configuration considered here, the slab does not affect the fields around the source ${ }^{3}$ and, therefore, $L_{11}=L_{22} \equiv L$, where $L$ is the self-inductance of the loops in free space. The nondiagonal terms of the inductance matrix

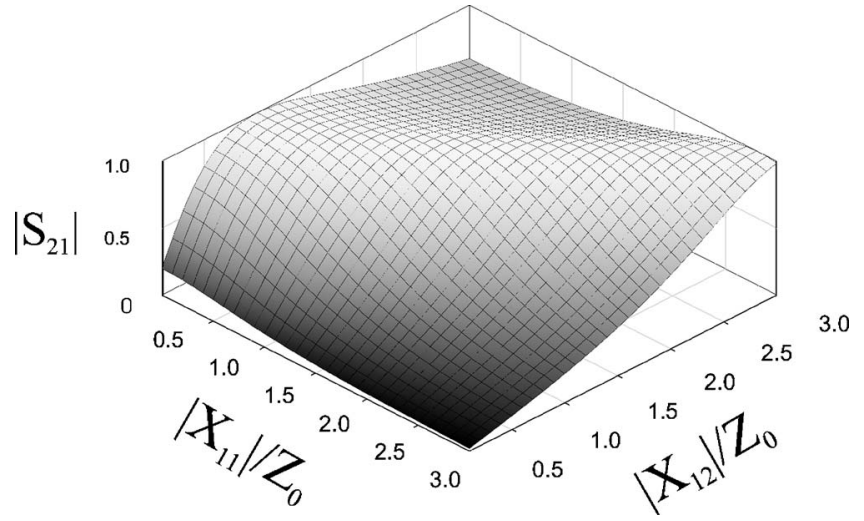

FIG. 4. Plot of the modulus of the transmission coefficient $t$ $=S_{21}$ as a function of the modulus of the reactances $X_{11}=X_{22}=$ $-\omega L$ and $X_{21}=-\omega M$.

account for the mutual inductance of the loop antennas in the presence of the slab, namely, $L_{12}=L_{21} \equiv M$. Thus, the transmission coefficient in (4) can be written as

$$
t=\frac{2 i \omega M Z_{0}}{\omega^{2}\left(L^{2}-M^{2}\right)+2 i \omega L Z_{0}-Z_{0}^{2}} .
$$

Since $L$ does not change with the position of the antennas, the spatial dependence in (5) comes only from $M$. The dependence of $|t|$ with the reactances $X_{11}=X_{22}=-\omega L$ and $X_{12}$ $=X_{21}=-\omega M$ deduced from (5) is shown in Fig. 4. It can be seen how the maximum transmission $(|t| \approx 1)$ is achieved when $X_{11} \simeq X_{12}$, except for very small values of the ratio $X_{11} / Z_{0}$. This effect is illustrated in Fig. 5, where the values of $X_{12}$ and $X_{11}$, which corresponds to a maximum of the transmission coefficient, are plotted. Therefore, except when $\omega L$ $\ll Z_{0}$, the transmission coefficient reaches its maximum at those points where $M \approx L\left(|t| \approx 1\right.$ if $\left.L \gtrsim Z_{0}\right)$. Since both the input and output antennas are identical, and the field at the source plane is reproduced at the image plane (see Fig. 3),

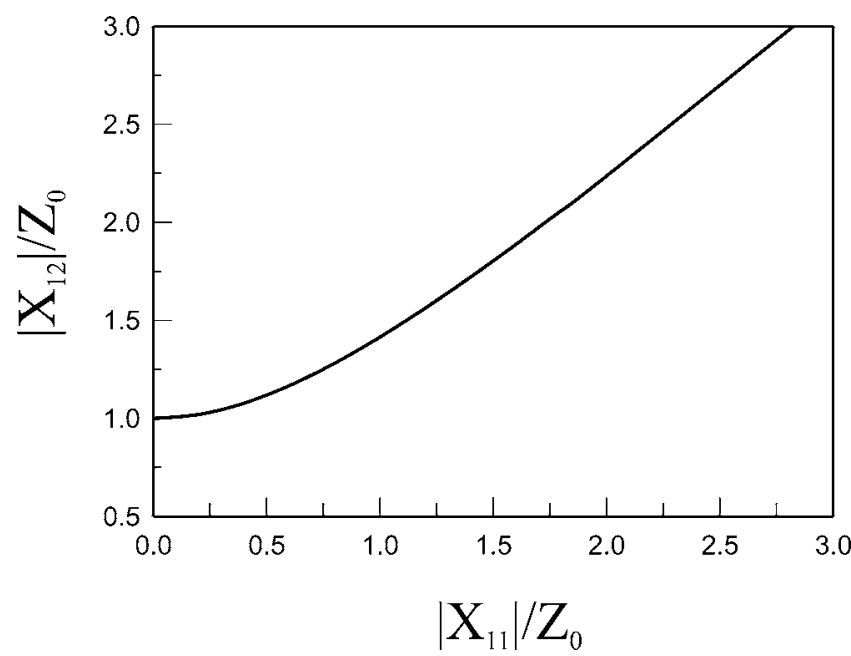

FIG. 5. Plot of the values of the reactances $X_{11}=X 22=-\omega L$ and $X_{112}=X 21=-\omega M$ for which the modulus of the transmission coefficient (5) reaches a maximum. 


\section{$\left|S_{21}\right|(d B)$}

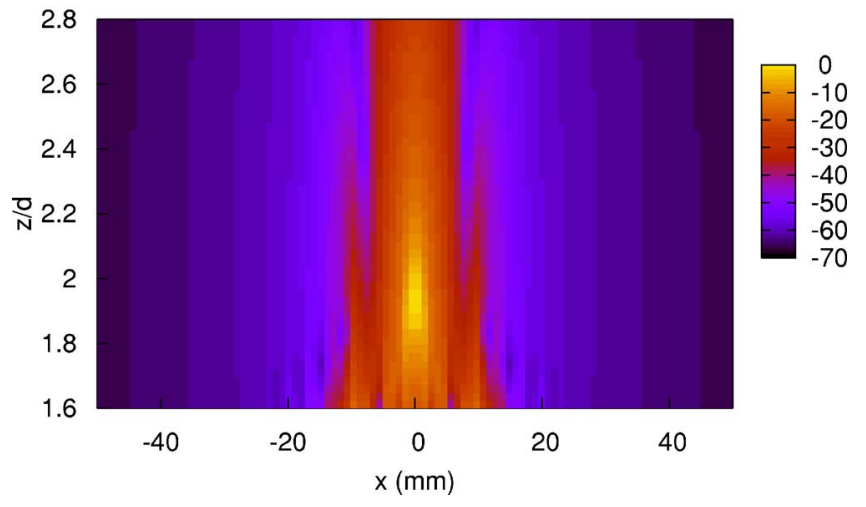

FIG. 6. (Color online) Map of the magnitude in decibels of the transmission coefficient, $\left|S_{21}\right|$, between the input and output antennas of Fig. 1. The structural parameters are as in Fig. 2. The output antenna is identical to the input one.

the condition $M \approx L$ is expected to be satisfied around the point $(x, y, z)=(0,0,2 d)$. In consequence, a maximum of the transmitted power should be detected around this particular point, and thus, it would appear as an "effective focusing point" of the perfect lens.

In order to show the above expected effects in a practical situation, the magnitude of the transmission coefficient has been numerically computed for the configuration under study. The impedance matrix has been calculated by imposing known currents, $I_{i}=1,0 \mathrm{~A}$, in the loops, and then computing the corresponding self and mutual inductances. The computation of the self-inductance is an standard electromagnetic problem, and the mutual inductance is obtained after computing the flux of the magnetic field given by (2) across the surface of the output antenna. The transmission coefficient is finally determined from (4), assuming $Z_{0}$ $=50 \Omega$. In Fig. 6, a map of the computed transmission coefficient for a system composed of two identical lossless loop antennas is shown. It can be observed that this figure substantially differs from Fig. 2; in particular, a clear maximum of $|t|$ can be observed in Fig. 6 in the neighborhood of the image at $(0,0,2 d)$. These results clearly show the difference between the transmitted power and the field distribution in the absence of the output antenna, and also how 3D-SRI can be obtained when the appropriate detector is used.

Let us now consider the measurement of the unperturbed field. For this purpose, the detector should be designed to affect the field distribution as little as possible. It could be closely achieved by loading the output antenna with an additional high resistance, which will significantly reduce the current induced at the output antenna, as well as its generated field. In Fig. 7 the computed values of the transmission coefficient along the $z$-axis of Fig. 1 are shown for different resistances, $R$, loading the output antenna. As is expected, the curve for $R=0 \Omega$ shows a maximum near the location of the image, which thus appears as a "focusing point" of the lens, at $z \approx 2 d$. (The small difference between the actual location of this maximum with respect to $z=2 d$ can be attributed to the not very high value of the ratio $\omega L / Z_{0} \approx 8$ in the case under study). As is also expected, the curves for the highest

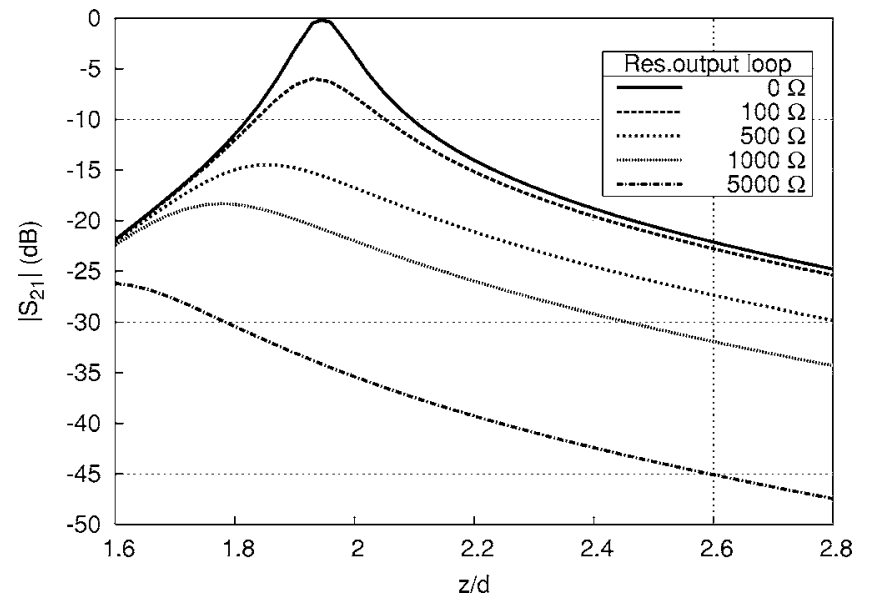

FIG. 7. Magnitude of the computed transmission coefficient along the $Z$ axis between the input and output antennas of Fig. 1 for different resistances loading the output antenna. The structural parameters are as in Fig. 2.

values of $R$ resemble the behavior of $\left|H_{z}\right|$ in Fig. 2 for the unperturbed configuration.

Another obvious strategy for reducing the perturbation of the field by the measurement is to reduce the radius of the output antenna of Fig. 1. This will reduce both $L_{2}$ (the inductance of the output antenna) and $M$ without changing $L_{1}$ (the inductance of the input loop). In the limit when $\omega L_{2}, \omega M$ $\ll Z_{0}$, (4) reduces to

$$
t \approx \frac{2 i \omega M}{i \omega L_{1}-Z_{0}}
$$

Since both $L_{1}$ and $Z_{0}$ do not depend on the location of the output, the measured transmission coefficient turns out to be proportional to $M$, i.e. to the magnetic field $H_{z}$ at the location of the output. Thus, the analyzed experimental setup is appropriate for the detection of the field of the unperturbed system formed by the input antenna and the lens. The magnitude of the transmission coefficient along the $z$ axis of Fig. 1 is plotted in Fig. 8 for several values of the radius of the output loop. The curves corresponding to $r_{2} \geqslant 4 \mathrm{~mm}$ clearly show that the location of the detected transmission maximum approaches the lens interface as the radius of the output loop is reduced. In the limit of small radius $\left(r_{2}=1 \mathrm{~mm}\right)$, the previously predicted monotonic increase of the field toward the right-hand-side lens interface is observed. Figure 8 also shows that the sensitivity of the experimental setup to small differences between the shapes of the input and output antennas is not very high. As it can be readily seen from the curves corresponding to $r_{2}=5$ and $4.5 \mathrm{~mm}$, the transmission peak is located very close to $2 d$ for moderate deviations of $r_{2}$ from its optimum value $r_{2}=r_{1}$.

\section{EXPERIMENT}

In order to show the application of the above theory to practical devices, the experimental demonstration of 3D-SRI recently reported by some of the authors ${ }^{11}$ will now be reexamined in light of the above considerations. Although the 


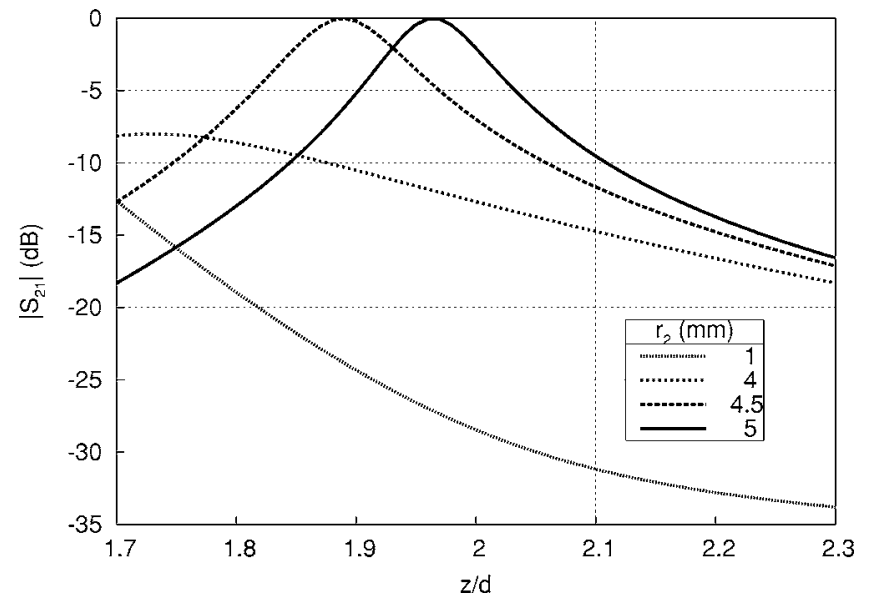

FIG. 8. Magnitude of the computed transmission coefficient along the $z$ axis between the input and output antennas of Fig. 1 for several values of the radius of the detector $\left(r_{2}\right)$. The structural parameters are as in Fig. 2.

underlying physics of the device analyzed there is not exactly the same as that of the left-handed perfect lens, ${ }^{11}$ both systems show the same process of amplification of evanescent modes and are equivalent in practice for the present purposes. Thus, experimental results plotted in Fig. 9 show the location of the maximum of the transmission coefficient along the $z$ axis of the magnetoinductive lens ${ }^{11}$ for different values of the resistance loading the output loop. The experimental setup used to obtain these results is described in detail in Ref. 11. The only difference is that the output antenna is now loaded by different microwave resistors to obtain the results shown in Fig. 9. These results show the same type of behavior as that reported in Fig. 7; namely, the location of a maximum for the magnitude of the transmission coefficient in the neighborhood of $z \approx 2 d$ for identical output and input antennas, and a similar displacement of the maximum of the transmission coefficient as the radius of the output antenna decreases. The agreement between the theory and the experiments can be considered as a validation of the present theory. It also shows that the ideas developed in the previous analysis are very general, and that, actually, they are applicable to any superresolution lens based on the amplification and amplitude restoration of FHs along the device.

\section{CONCLUSIONS}

Superresolution in metamaterial superlenses has been investigated. As is well known, this imaging is primarily due to the amplification of evanescent modes inside the lens. As a consequence, superresolution in the planes parallel to the slab is unavoidably compensated by a drastic loss of resolution in the direction perpendicular to the slab. However, since evanescent modes do not carry power, any physical detection (namely, a measurement) of the image at the back side of the lens will require the existence of some amount of

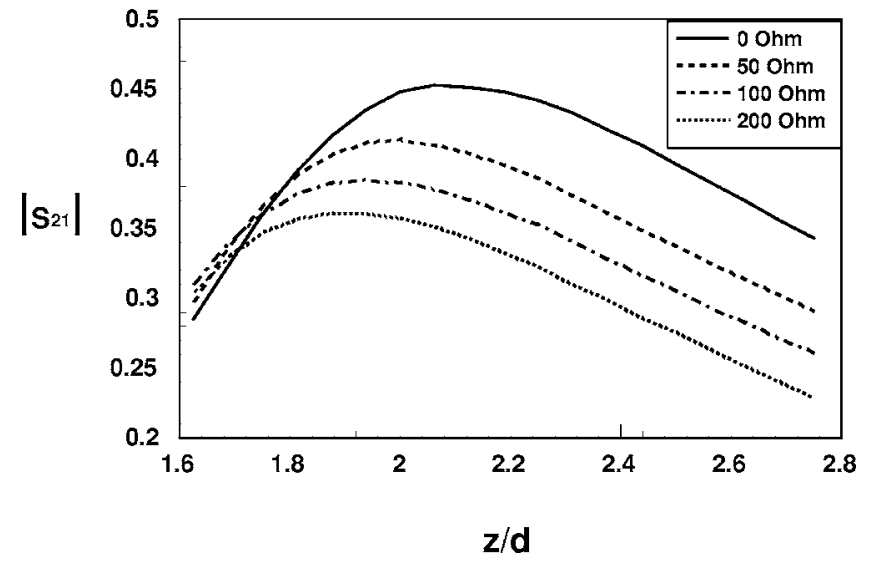

FIG. 9. Plots of the measured magnitude of the transmission coefficient between the input and output antennas of Fig. 1 when the slab is substituted by the magnetoinductive lens reported in Ref. 11. (Frequency $=3 \mathrm{GHz}$ ).

transmitted power from the source to the detector, which can significantly affect the original field distribution. If this last effect is taken into account, the secondary fields generated by the presence of the detector should be considered. Following this approach, it has been shown that the transmission coefficient between the source and the detector can be increased considerably. Provided that the appropriate detector is employed (typically, a lossless output antenna identical to the input antenna), this maximum will occur in a neighborhood of the image, thus resulting in superresolution imaging also in the direction perpendicular to the slab. However, it should be emphasized that for obtaining this 3D-SRI, some previous knowledge of the source is necessary in order to design the detector properly. Thus, a general conclusion arises from the analysis: Superresolution in metamaterial superlenses is always incomplete; if the distance from the source to the lens is known, the shape and characteristics of the source can be recovered without uncertainty from the analysis of the field distribution at the image side of the lens. Conversely, if the shape and characteristics of the source are known, it is possible to design an appropriate detector in order to localize the source spatially from the values of the transmission coefficient between the source and the detector. However, it is impossible to determine, simultaneously, by means of a metamaterial superlens, the location, shape, and characteristics of an unknown source with a resolution overcoming the diffraction limit. We feel that the reported analysis and experiments, as well as the conclusions arising from them, will be of importance in the design of metamaterial superresolution devices.

\section{ACKNOWLEDGMENT}

This work has been supported by the Spanish Ministry of Education and Science by project Contract No. TEC200404249-C02-02. 
${ }^{1}$ V. G. Veselago, Sov. Phys. Usp. 10, 509 (1968).

${ }^{2}$ A. A. Houck, J. B. Brock, and I. L. Chuang, Phys. Rev. Lett. 90, 137401 (2003).

${ }^{3}$ J. B. Pendry, Phys. Rev. Lett. 85, 3966 (2000).

${ }^{4}$ A. Grbic and G. V. Eleftheriades, Phys. Rev. Lett. 92, 117403 (2004).

${ }^{5}$ A. N. Lagarkov and V. N. Kissel, Phys. Rev. Lett. 92, 077401 (2004).

${ }^{6}$ N. Fang and X. Zhang, Appl. Phys. Lett. 82, 161 (2003).

${ }^{7}$ N. Fang, H. Lee, C. Sung, and X. Zhang, Science 308, 534 (2005).

${ }^{8}$ R. J. Blaikie and D. O. S. Melville, J. Opt. A, Pure Appl. Opt. 7, S176 (2005).

${ }^{9}$ R. Marqués, F. Mesa, and F. Medina, Appl. Phys. Lett. 86, 023505 (2005).
${ }^{10}$ S. Maslowski, S. Tretyakov, and P. Alitalo, J. Appl. Phys. 96, 161 (2004).

${ }^{11}$ M. Freire and R. Marqués, Appl. Phys. Lett. 86, 182505 (2005).

${ }^{12}$ J. D. Baena, L. Jelinek, R. Marqués, and F. Medina, Phys. Rev. B 72, 075116 (2005).

${ }^{13}$ N. García and M. Nieto-Vesperinas, Phys. Rev. Lett. 88, 207403 (2002).

${ }^{14}$ D. R. Smith, D. Schurig, M. Rosenbluth, S. Schultz, S. Anantha Ramakrishna, and J. B. Pendry, Appl. Phys. Lett. 82, 1506 (2003).

${ }^{15}$ R. Marqués and J. D. Baena, Microwave Opt. Technol. Lett. 41, 290 (2004).

${ }^{16}$ V. G. Veselago, cond-mat/0501438 (unpublished).

${ }^{17}$ D. M. Pozar, Microwave Engineering (Wiley, New York, 1998), 2nd ed. 